\title{
Adición de un cortocircuito arterio-venoso durante el soporte vital extracorpóreo veno-arterial de un paciente con síndrome cardiopulmonar por Hantavirus. Caso clínico
}

\author{
Vinko Tomicic F, Mauricio Espinoza R, Javier Torres M, \\ Juan Abarca Z, José Miguel Montes S, Mario Luppi N, \\ Andrés Concha F, Enrique Reynolds H, Alberto Laporte M, \\ Claudio Canals L, Pablo Vial C. \\ Addition of an arterio-venous shunt \\ during veno-arterial extracorporeal \\ life support in a patient with \\ Hantavirus pulmonary syndrome
}

A subgroup of patients infected with the Hantavirus develops a pulmonary syndrome (HPS) characterized by severe acute respiratory failure and myocardial depression, that has a high mortality rate. Extracorporeal life support (ECLS) could be a valuable therapeutic tool in such patients. We report a 24 years old male with HPS that was successfully managed when an arterio-venous shunt was added to a conventional veno-arterial ECLS technique. Precise criteria have been developed to predict which patients should be considered for this treatment (Rev Méd Chile 2005; 133: 817-22).

(Key Words: Extracorporeal circulation; Hantavirus; hantavirus pulmonary syndrome)

Recibido el 21 de septiembre, 2004. Aceptado el 2 de mayo, 2005.

Departamento de Paciente Crítico, Clínica Alemana de Santiago. Facultad de Medicina,

Clínica Alemana-Universidad del Desarrollo. Santiago de Chile.

$E^{1}$ soporte vital extracorpóreo veno-arterial (ECLS/V-A) es una alternativa terapéutica para aquellos pacientes que cursan con falla cardiopul-

Correspondencia a: Vinko Tomicic Flores. Av. Vitacura 5951 - Santiago. Teléfonos: 2101600-2101409. Fax: 2101068-2101411. E mail: vinkotomicic@udd.cl monar que, a pesar de optimizar la ventilación mecánica y el soporte hemodinámico o aplicar terapias avanzadas (ventilación diferencial, posición prono, óxido nítrico, etc), son incapaces de superar la hipoxemia y la depresión miocárdica ${ }^{1,2}$.

El síndrome cardiopulmonar por virus Hanta (SCPH) se caracteriza por hipotensión, disminución del índice cardíaco, aumento de la resistencia 
vascular sistémica y edema pulmonar por incremento de la permeabilidad vascular. En un subgrupo de enfermos, estas manifestaciones pueden culminar en falla respiratoria y depresión miocárdica severas, asociadas a elevada mortalidad ${ }^{3-5}$. En estos casos, el ECLS/V-A puede sustituir temporalmente la función cardíaca y respiratoria mientras se supera el período de máximo riesgo vital ${ }^{6}$.

Se presenta el caso de un paciente con $\mathrm{SCPH}$, tratado con esta técnica, que durante las primeras horas mostró mejoría parcial, manteniendo coloración cianótica del hemicuerpo superior y persistencia del láctico arterial elevado. Este hecho se relaciona con la localización de la cánula de retorno arterial, la cual determina la distribución de la sangre oxigenada en la circulación sistémica ${ }^{7}$. Se describe la adición de un cortocircuito de sangre oxigenada, hacia la vena cava superior como alternativa para superar esta condición ${ }^{8}$.

\section{CASO CLÍNICO}

Varón de 24 años, previamente sano, inició compromiso de estado general, fiebre, tos seca y diarrea en Colbún (VII región). Tres días después, la radiografía de tórax mostró infiltrado basal izquierdo discreto y los exámenes de laboratorio: hematocrito de 46\%, 6.100 glóbulos blancos, 16\% de baciliformes, 92.000 plaquetas, SGOT 133 U/L y TTPA 79 s. Al cuarto día se agregó dificultad respiratoria y progresión de los infiltrados radiológicos pulmonares.

Ingresó a UCI consciente, hipotenso, taquicárdico, taquipneico, febril con APACHE II de 27 puntos $^{9}$ y altos requerimientos de oxígeno. Se instaló catéter de arteria pulmonar (CAP) y monitor PiCCO (Pulsion Medical System, Munich, Germania) cuyos resultados sugirieron edema pulmonar de permeabilidad ${ }^{10,11}$. Seis horas después se conectó a ventilación mecánica (Tabla 1).

A las 29 h, su situación cardiorrespiratoria se deterioró, a pesar del aumento progresivo del soporte vasoactivo y optimización de la ventilación mecánica procurando un impacto hemodinámico mínimo ${ }^{12}$. Tabla 1.

Con estos parámetros se decidió conexión a soporte cardiopulmonar extracorpóreo vía venoarterial fémoro-femoral, con cánulas 21 y 19 French (Biomedicus, Medtronic, Grand Rapids, MI, USA) bajo anticoagulación sistémica.

Tabla 1. Parámetros hemodinámicos, respiratorios e indicadores de perfusión

\begin{tabular}{|c|c|c|c|c|c|c|c|}
\hline \multirow[t]{2}{*}{ Parámetros } & \multirow{2}{*}{$\begin{array}{c}\text { Ingreso } \\
\text { UCI }\end{array}$} & \multirow{2}{*}{$\begin{array}{c}\text { Ingreso } \\
\text { ECLS }\end{array}$} & \multirow{2}{*}{$\begin{array}{c}\text { ECLS } \\
\text { V-A }\end{array}$} & \multicolumn{2}{|c|}{ ECLS Mixto } & \multirow{2}{*}{$\begin{array}{l}\text { Post } \\
\text { ECLS }\end{array}$} & \multirow{2}{*}{$\begin{array}{c}\text { Valores } \\
\text { normales }\end{array}$} \\
\hline & & & & $2 \mathrm{~h}$ & $48 \mathrm{~h}$ & & \\
\hline $\mathrm{IC}\left(\mathrm{L} / \mathrm{min} / \mathrm{m}^{2}\right)$ & 2,22 & 1,69 & - & - & - & 4,95 & $2,5-4$ \\
\hline RVSI (dvn*seg $\left.{ }^{*} \mathrm{~m}^{2} / \mathrm{cm}^{5}\right)$ & 3.786 & 2.321 & - & - & - & 1.098 & $1.200-2.500$ \\
\hline RVPI (dyn* seg $\left.^{*} m^{2} / \mathrm{cm}^{5}\right)$ & 361 & 663 & - & - & - & 162 & \\
\hline PAoP $(\mathrm{mmHg})$ & 8 & 17 & 19 & 16 & 15 & 21 & $5-12$ \\
\hline Qs/Qt (\%) & 15,4 & 38,8 & - & - & - & 8,7 & $<10$ \\
\hline ITVBI $\left(\mathrm{mL} / \mathrm{m}^{2}\right)$ & 535 & 572 & - & - & - & - & $850-1.000$ \\
\hline EVLWI (mL/kg) & 14 & 16 & - & - & - & - & $3-7$ \\
\hline $\mathrm{PaO}_{2} / \mathrm{FiO}_{2}$ & 218 & 41 & 48 & 71 & 147 & 392 & $>400$ \\
\hline Iox & 9 & 43,9 & 35 & 28 & 16 & 5 & $<2$ \\
\hline $\mathrm{SaO}_{2}(\%)$ & 97 & 64 & 75 & 93 & 99 & 99 & $95-99$ \\
\hline $\mathrm{pH}$ arterial & 7,29 & 7,18 & 7,24 & 7,31 & 7,30 & 7,43 & $7,37-7,43$ \\
\hline $\mathrm{SvO}_{2}(\%)$ & 66 & 41 & 58 & 76 & 79 & 71 & $68-77$ \\
\hline Lactato arterial (mMol/L) & 3 & 17 & 12 & 6 & 6 & 5 & $<2,4$ \\
\hline
\end{tabular}

IC: índice cardíaco; RVSI: resistencia vascular sistémica indexada; RVPI: resistencia vascular pulmonar indexada; PAoP: presión arterial de oclusión pulmonar, Qs/Qt: cortocircuito intrapulmonar (shunt); ITBVI: volumen sanguíneo intratorácico indexado. EVLWI: agua pulmonar extravascular indexada. Iox: índice de oxigenación = Presión media vía aérea x $100 / \mathrm{PaO}_{2} / \mathrm{FiO}_{2} ; \mathrm{SaO}_{2}$ : saturación arterial; $\mathrm{Sv}_{2}$ : saturación venosa mixta. 
Circuito veno-arterial. El drenaje venoso se realizó desde la aurícula derecha hacia una bomba centrífuga magnética (Sarns Inc/3M, Ann Arbor, Mich.) y oxigenador de fibras perforadas (Affinity NT, Medtronic, Minneapolis, MN, USA). La sangre oxigenada retornaba a la aorta descendente vía arteria femoral izquierda. El flujo de la bomba se inició a 3,8 L/min y se aumentó progresivamente hasta alcanzar 5,3 L/min a las $12 \mathrm{~h}$ (Figura 1). El $\mathrm{pH}$ arterial, $\mathrm{SaO}_{2}$ y $\mathrm{SvO}_{2}$ mejoraron, pero el hemicuerpo superior permaneció cianótico, motivo por el cual se decidió derivar sangre oxigenada hacia el sistema venoso central. Tabla 1.

Circuito veno-arterial/arterio-venoso (ECLS/VA$\mathrm{AV})$. Se colocó un catéter doble lumen $11 \mathrm{~F}$ en la vena yugular interna izquierda. Ambos lúmenes recibieron sangre post-oxigenador con un flujo sanguíneo de $300 \mathrm{ml} / \mathrm{min}$ en cada lumen. Debido a insuficiencia renal aguda, la terapia de reemplazo renal continuo se interpuso en uno de los lúmenes (Figura 2). Se constituyó así el cortocircuito mixto, luego del cual el paciente mejoró gradualmente permitiendo, hacia el tercer día, reducir progresivamente la $\mathrm{FiO}_{2}$ hasta $30 \%$, retirar la infusión continua de adrenalina y reducir la norepinefrina a $0,1 \mu \mathrm{g} / \mathrm{kg} / \mathrm{min}$. Al cuarto día el flujo de la bomba se encontraba en $3 \mathrm{~L} / \mathrm{min}$ y la dobutamina a $10 \mu \mathrm{g} / \mathrm{kg} / \mathrm{min}$ como único apoyo vasoactivo. A las $102 \mathrm{~h}$ se retiró el cortocircuito A$\mathrm{V}$, momento en que se introdujo una infusión de nitroglicerina para controlar el incremento de la presión arterial pulmonar. El paciente se mantuvo con el flujo de la bomba en $3 \mathrm{~L} / \mathrm{min}$ por $2 \mathrm{~h}$. Posteriormente el flujo se redujo en $1 \mathrm{~L} /$ min cada dos horas hasta suspenderlo. Por precaución, la bomba se dejó recirculando en una línea extracorpórea (puente) por una hora, luego de la cual se detuvo y se procedió al retiro de las cánulas después de 110 h en ECLS. Siete días más tarde, el paciente fue extubado y se dió el alta de UCI a los 21 días. Tabla 1.

\section{DISCUSIÓN}

El soporte circulatorio extracorpóreo está indicado en pacientes que cursan con falla respiratoria aguda potencialmente reversible, que poseen riesgo vital elevado y no responden a terapia conven-

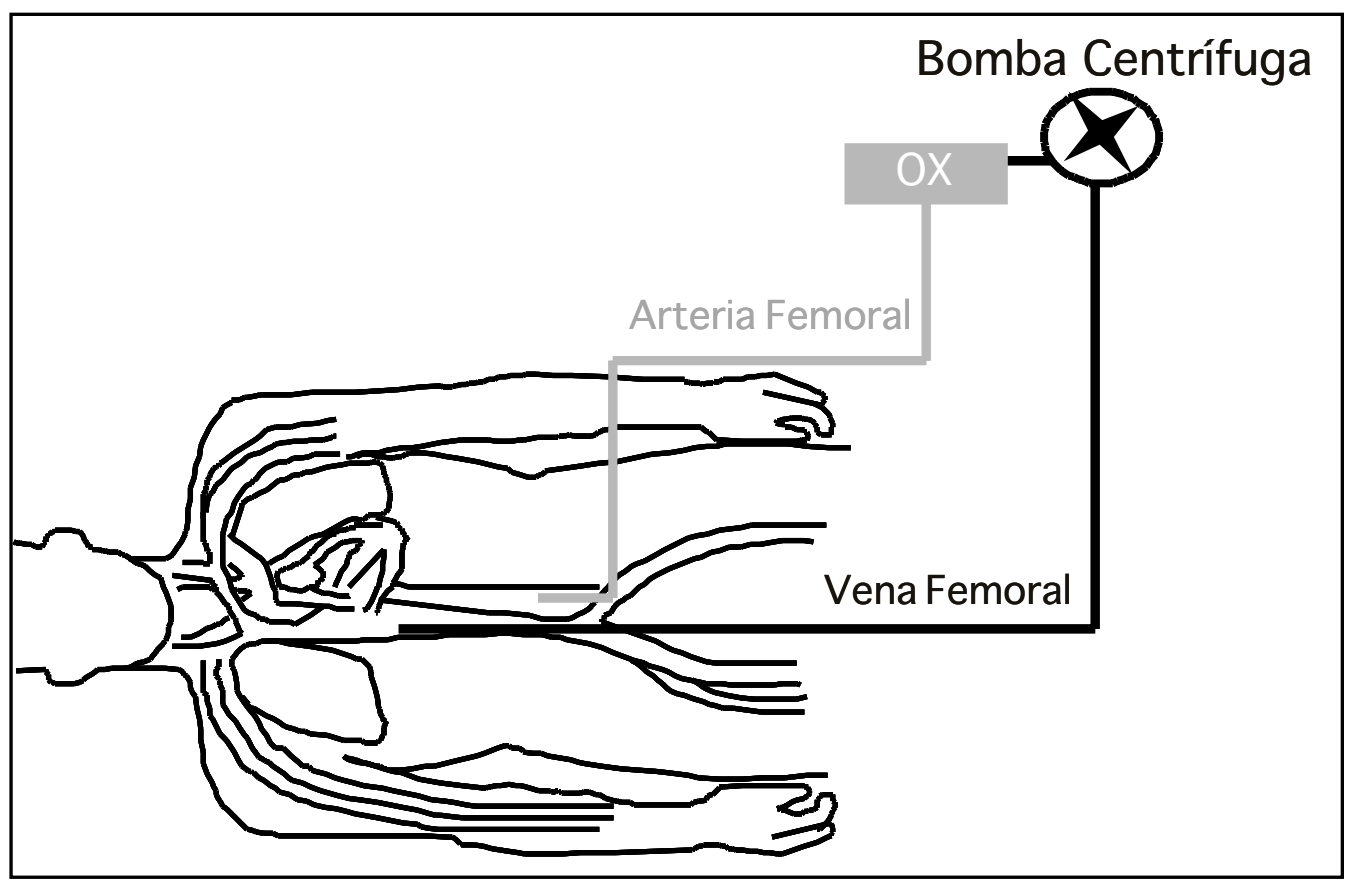

Figura 1. Esquema de la conexión del paciente a ECLS/V-A. 


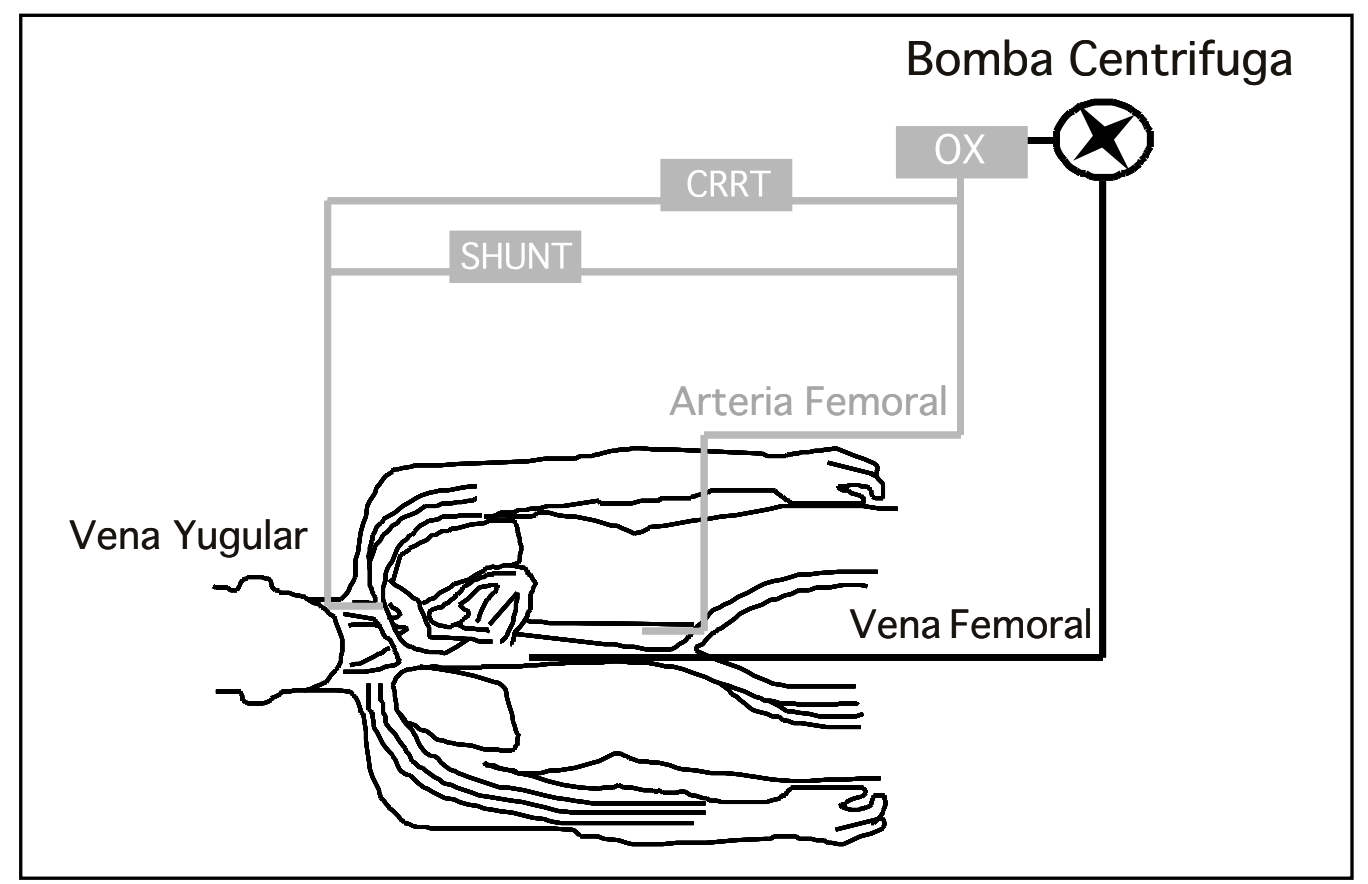

Figura 2. Cortocircuito veno-arterial/arterio-venoso mixto. CRRT: Terapia de reemplazo continuo de la función renal; SHUNT: cortocircuito arterio-venoso; OX: oxigenador.

cional. En este grupo destacan el síndrome de distress respiratorio agudo, disfunción del injerto postrasplante pulmonar y edema pulmonar postneumonectomía. Existen algunos criterios para decidir la conexión a ECLS (cortocircuito intrapulmonar $>30 \%$ con $\mathrm{FiO}_{2}>0,6$, distensibilidad $<0,5$ $\mathrm{ml} / \mathrm{cmH}_{2} \mathrm{O} / \mathrm{kg}$, hipencarbia incorregible con $\mathrm{pH}$ $<7,0$ y presión inspiratoria pico $>40 \mathrm{cmH}_{2} \mathrm{O}$ ), sin embargo, el juicio clínico y el análisis caso a caso pueden influir en la decisión. En adultos, esta técnica no ha tenido la eficacia que ha mostrado en el escenario neonatal, sin embargo, siempre debe ser considerada como una herramienta más en pacientes cuya mortalidad estimada es mayor a $70 \%$ y poseen una probabilidad razonable de recuperación pulmonar ${ }^{1,13,14}$.

El ECLS ofrece dos formas básicas de soporte: veno-venoso (ECLS/V-V) cuando el circuito se origina y termina en el compartimiento venoso y veno-arterial (ECLS/V-A) cuando se inicia en el lecho venoso y termina en el arterial ${ }^{1,2,7}$.

El ECLS/V-V, al establecer un cortocircuito restringido al compartimiento venoso de retorno, posee un efecto mínimo sobre la función cardíaca y una mejoría en esta última sólo puede ser atribuida a elevación de la saturación arterial. Esta forma de soporte, por lo tanto, está indicada exclusivamente cuando la función cardíaca es suficiente para suplir la perfusión tisular. En general, ambas cánulas venosas se sitúan próximas a la aurícula derecha, de modo que parte de la sangre oxigenada reingresa en el circuito. Esto último explica por qué incrementos del flujo extracorpóreo superiores a $50 \%$ del gasto cardíaco nativo, no ofrecen ventajas en la oxigenación sistémica. Este fenómeno, conocido como recirculación, limita la capacidad para elevar la saturación arterial a valores sólo discretamente superiores a $80 \mathrm{u} 85 \%$, hecho que hace indispensable mantener el soporte ventilatorio. En esta variedad de ECLS la presión arterial y el monitoreo hemodinámico con CAP no se alteran sustancialmente $^{2,7}$.

En el ECLS/V-A, en cambio, se establece un cortocircuito entre el compartimiento venoso y la circulación arterial sistémica, por lo que sólo una 
parte del retorno venoso sistémico ingresa a la circulación pulmonar nativa. Esto permite minimizar el cortocircuito intrapulmonar y reducir la presión arterial pulmonar, mecanismo que descomprime el ventrículo derecho y optimiza la función cardíaca. El flujo extracorpóreo retorna sangre completamente oxigenada a la circulación sistémica elevando sustancialmente la $\mathrm{PaO}_{2}$, lo que hace posible reducir el soporte ventilatorio, características que lo sitúan como el método de elección en pacientes que cursan con deterioro concomitante de la función cardíaca y pulmonar, como es el caso del paciente aquí presentado. Debemos considerar, además, que al existir un cortocircuito de derecha a izquierda, tanto las variables entregadas por el CAP, como la presión arterial de pulso carecen de significado clínico y la presión arterial media pasa a ser el principal parámetro de monitorización ${ }^{2,7,15,16}$

En este tipo de circuito, la post-carga ventricular izquierda depende en gran medida de la activación del eje renina-angiotensina aldosterona, magnitud del flujo extracorpóreo seleccionado y de la localización de la cánula arterial ${ }^{17}$. Cuando ésta se ubica en el arco aórtico y el flujo extracorpóreo debe ser elevado para satisfacer las necesidades del paciente, se puede producir un incremento desmedido de la post-carga ventricular izquierda, motivo por el cual en nuestro paciente, la cánula arterial se instaló a nivel infradiafragmático. Cabe destacar que con esta localización, la sangre oxigenada se mezcla con aquella que posee bajo contenido arterial de oxígeno proveniente de la circulación cardiopulmonar nativa, por lo tanto, dicha mezcla se distribuye a través del hemicuerpo inferior antes de retornar a la circulación pulmonar y alcanzar el hemicuerpo superior, hecho que explica la coloración cianótica en dicho territorio 7,8 (Figura 1). En estas circunstancias el contenido arterial de oxíge-

\section{REFERENCIAS}

1. LEWANDOWSKY $\mathrm{K}$, LOHBRUNNeR $\mathrm{H}$, LeWANDOWSKY $\mathrm{M}$, FALKE K. Outcome and follow-up of adults following extracorporeal life support. En Zwischenberger J, Steinhorn R Bartlett R, ed. Extracorporeal cardiopulmonary support in critical care. Second no en el hemicuerpo superior y especialmente en el cerebro, depende de la magnitud del cortocircuito intrapulmonar el cual, en nuestro paciente, pudo ser subestimado en el momento de instalar el ECLS/V-A, ya que el gasto cardíaco preconexión se encontraba muy disminuido ${ }^{18}$.

Para superar este problema, es posible derivar sangre ya oxigenada hacia el sistema venoso central (cortocircuito A-V) o adicionar una segunda cánula arterial en las cercanías del arco aórtico (Ej: arteria axilar), alternativa que requiere manejo quirúrgico ${ }^{7}$. Considerando la necesidad de un catéter doble lumen para iniciar el reemplazo de la función renal, optamos por la instalación de un puente venoso hacia la vena yugular intema, con lo cual conseguimos elevar el contenido de oxígeno venoso mixto y corregir la cianosis del hemicuerpo superior. Tabla 1.

Los mejores resultados obtenidos con el uso de ECLS en los últimos años, se relacionan con el perfeccionamiento de la técnica y modificación de los criterios de selección de los pacientes ${ }^{14}$. Entre ellos destaca la implementación precoz del ECLS, es decir, antes que se instale el daño pulmonar inducido por ventilación mecánica ${ }^{19}$.

En nuestro país, la mortalidad del SCPH sigue siendo elevada (29\%) y aquellos enfermos que en las primeras $24 \mathrm{~h}$ desarrollan hipoxemia refractaria y disfunción cardíaca de progresión rápida, tienen $100 \%$ de mortalidad ${ }^{3,20}$. Para identificar pacientes con SCPH que requerirán asistencia extracorpórea se propone la presencia de shock refractario con índice cardíaco $<2,5 \mathrm{~L} / \mathrm{min} / \mathrm{m}^{2}$ y láctico sérico $>4$ $\mathrm{mmol} / \mathrm{L}^{6,21}$.

El SCPH se presenta en pacientes jóvenes habitualmente sin comorbilidad, posee un período de riesgo vital elevado, pero breve y autolimitado, elementos que justifican plenamente el uso de esta técnica en aquellos enfermos que adoptan un curso catastrófico.

Edition: Editorial Extracorporeal Life Support Organization, 2000; 531-62.

2. Moulton S, Delius R, Arensman R. Vascular access for extracorporeal life support. En Arensman R, Cornish J, ed. Extracorporeal life support. Editorial Blackwell Scientific Publications. 1993; 175-94. 
3. Castimo C, Naranjo J, Sepúlveda A, Ossa G, Levy H. Hantavirus pulmonary syndrome due to Andes virus in Temuco, Chile. Clinical experience with 16 adults. Chest 2001; 120: 548-54.

4. Hawn G, Simpson S, Crowell R, James DS, Koster FT, MERTZ GJ ET AL. Cardiopulmonary manifestations of hantavirus pulmonary syndrome. Crit Care Med 1996; 24: 252-8.

5. Duchin JS, Koster FT, Peters CJ, Simpson GL, TEMPEST B, KAKI SR ET AL. Hantavirus pulmonary syndrome: A clinical description of 17 patients with a newly recognized disease. NEJM 1994; 330: 949-55.

6. Crowley M, Katz RW, Kessler R, Simpson SQ, Levy H, HaLiN GW ET aL. Successful treatment of adults with severe Hantavirus pulmonary syndrome with extracorporeal membrane oxygenation. Crit Care Med 1998; 26: 409-14.

7. Dalton HJ. Extracorporeal life support in the new millennium: Forging ahead or fading out? New Horiz 1999; 7: 414-32.

8. Hill JD, Ratuff JL, Falat RJ, Tucker hj, Lamy M, Dietrich H-P et al. Prognostic factors in the treatment of acute respiratory insufficiency with long-term extracorporeal oxygenation. J Thoracic Cardiovascular Surgery 1974; 68: 905-17.

9. Knaus W, Draper E, Wagner D, Zimmerman J. APACHE II: a severity of disease classification system. Crit Care Med 1985; 13: 818-29.

10. Tomicic V, Espinoza M, Hernández G, Abarca L. Monitoreo de la volemia y el agua pulmonar extravascular en UCI. Medicina Intensiva 2002; 17: 15-9.

11. Romero C, Andresen M, Díaz O, Tomicic V, Barahona F, Mercado M et al. Síndrome cardiopulmonar por Hantavirus: utilidad de la monitorización con el sistema PiCCO. Rev Méd Chile 2003; 131: 1173-8.

12. Tomicic V, Andresen M, Romero C, Mercado M. Impacto hemodinánico de la presión positiva de fin de espiración (PEEP) durante la falla respiratoria grave: visión actual. Rev Méd Chile 2002; 130: 1419-30.

13. Applications and management of ECMO. En Meyer D, Jessen M, ed. Extracorporeal life support. Editorial Landes Bioscience, 2001; 84-97.

14. Kola S, Awad S, Rich P, Schreiner R, Hirschl R, BARTLETT R. Extracorporeal life support for 100 adult patients with severe respiratory failure. Ann Surg 1997; 226: 544-66.

15. Tomicic V, CASTILO L Soporte extracorpóreo con membrana de oxigenación (ECMO). En Vial PA, Valdivieso F, Ferrés M, ed. Manejo del paciente crítico con síndrome cardiopulmonar por hantavirus. Primera Edición: Editorial Salesianos S.A., 2004; 75-83.

16. Castilo L, Velasco S, Irarrázabal MJ, Garayar B, Hernández G, Córdova S et al. Falla cardiorrespiratoria severa tratada con asistencia cardiopulmonar extracorpórea. Caso Clínico. Rev Méd Chile 2002; 130: 545-50.

17. Hines M, Fauza D, Wilson J. ECMO: Hemodynamics, perfusion and blood volume. En Zwischenberger J, Steinhorn R Bartlett R, ed. Extracorporeal cardiopulmonary support in critical care. Second Edition: Editorial Extracorporeal Life Support Organization, 2000; 97-112.

18. Lynch JP, Mhyre JG, DANTZKer DR. Influence of cardiac output on intrapulmonary shunt. J Appl Physiol 1979; 46: 315-21.

19. Pranikoff T, Hirschl R, Steimle C, Anderson $H$, BARTLETT R. Mortality is directly related to the duration of mechanical ventilation before the initiation of extracorporeal life support for severe respiratory failure. Crit Care Med 1997; 25: 28-32.

20. http://epi.minsal.cl/epi/html/frames/frame2.htm

21. Serna D, BRenNeR M, Chen JC. Severe hantavinus pulmonary syndrome: A new indication for extracorporeal life support? Crit Care Med 1998; 26: 217-8. 